Sociologie et sociétés

\title{
Dépendance économique, subordination politique et oppression nationale
}

\section{Le Québec 1960-1977}

\section{Economic Dependancy, Political Subordination and National Oppression} Quebec 1960-1977

\section{Paul R. BÉLANGER et Céline SAINT-PIERRE}

Volume 10, numéro 2, octobre 1978

Changement social et rapports de classes

URI : https://id.erudit.org/iderudit/001039ar

DOI : https://doi.org/10.7202/001039ar

Aller au sommaire du numéro

\section{Éditeur(s)}

Les Presses de l'Université de Montréal

ISSN

0038-030X (imprimé)

1492-1375 (numérique)

Découvrir la revue

Citer cet article

BÉLANGER, P. R. \& SAINT-PIERRE, C. (1978). Dépendance économique, subordination politique et oppression nationale : le Québec 1960-1977. Sociologie et sociétés, 10(2), 123-148. https://doi.org/10.7202/001039ar

\section{Résumé de l'article}

Partant d'un bref commentaire critique du concept de mouvement social dans le schéma tourainien, ce qui constitue la première partie de cet article, nous tentons, dans la seconde partie, de mettre en relief l'articulation qui existe entre la lutte nationale et la lutte des classes au Québec. Il s'agit donc d'identifier les multiples contradictions dont est traversé le Québec en procédant d'abord à un rappel historique rapide et en tentant par la suite de définir la place qu'y occupe l'oppression nationale. Nous posons, dans la troisième partie, la questions des effets spécifiques de l'oppression nationale sur la bourgeoisie, la petite-bourgeoisie et la classe ouvrière. Dans la dernière partie, l'analyse de la conjoncture de la période 1960-1977 nous permet de cerner de plus près les rapports de classe et les enjeux qui marquent leur dynamique. 


\section{Dépendance économique subordination politique et oppression nationale : le Québec 1960-1977*}

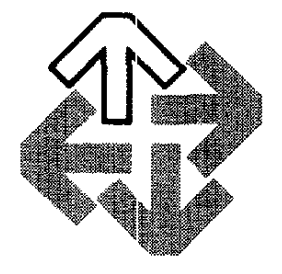

PAUl R. BELANGer et CÉline SAINT-PIERRE

Les analyses de la conjoncture qui ont été produites sur la société québése oscillent entre deux types d'approche : pour les uns, l'histoire du Québec s'est faite à travers les luttes nationales et le nationalisme est l'expression directe de cette dynamique historique; pour les autres, cette dynamique relève principalement de la lutte des classes et la question nationale est à peine esquissée daris la problématique.

Dans le premier cas, la problématique ne s'appuie aucunement sur la lutte des classes comme schéma explicatif et dans le second cas, ce sont les luttes nationales qui subissent le même sort. Dans les deux approches, les luttes de classes et les luttes nationales sont dissociées.

Nous croyons, quant à nous, que les deux problématiques sont erronées et qu'il faille absolument lier les deux facteurs pour rendre compte de la spécificité du Québec dans son développement historique. En ce sens, nous nous proposons dans cet article de présenter une analyse, bien sommaire, nous en sommes conscients, qui tente de rompre avec ces deux approches. Pour ce faire nous allons, dans un premier temps, introduire notre sujet par une brève

* Ce texte a été écrit au printemps 1976 puis revisé pour publication à l'automne 1977. Notre analyse de la conjoncture est donc dépendante du moment où nous avons écrit ce texte. 
critique des rapports entre classes et luttes nationales tels que présentés par Alain Touraine dans son livre les Sociétés dépendantes.

Dans le seconde et la troisième parties du texte, nous tenterons de mettre en relief l'articulation qui existe entre la lutte nationale et la lutte des classes et nous terminerons par une quatrième partie portant sur la conjoncture récente au Québec.

\section{MOUVEMENT SOCIAL OU LUTTE DE CLASSES}

Dans ses essais sur l'Amérique latine, Alain Touraine soutient qu'il est nécessaire d'utiliser trois principes d'analyse : mobilité sociale, nationalisme et rapports de classes pour rendre compte de la complexité et de la dynamique des sociétés dépendantes. Dans les sociétés dépendantes, selon lui, ce ne sont pas tellement les classes sociales qui s'affrontent qui occupent le champ des luttes sociales mais des mouvements sociaux de développement caractérisés par leur multi-dimensionalité. "L'expérience européenne nous a convaincus qu'un mouvement social était d'autant plus important et central qu'il était plus unidimensionnel, c'est-à-dire qu'il remettait en cause plus directement les rapports des classes. Dans les sociétés dépendantes, un mouvement dominé par la lutte des classes, par la volonté de libération nationale ou par un effort de modernisation économique et sociale est condamné à ne pas être important... Seule la combinaison de ces trois dimensions : lutte de classes, libération nationale, modernisation sociale, permet de constituer un mouvement important ${ }^{1}$.» Cette distinction lui permet de caractériser des types de mouvements sociaux (révolte populaire, populisme, mouvements nationaux populaires, etc.) selon la place accordée à l'un ou l'autre des éléments par chacun des mouvements.

Cette problématique semble au premier abord d'une grande efficacité car elle permet d'identifier les «aspects les plus divers de la vie nationale ${ }^{2}$ » en même temps qu'elle indique des types de liaison, par les mouvements sociaux, des différents enjeux des luttes sociales. Toutefois, et sans entrer dans une critique épistémologique de la place du concept de mouvement social dans la théorie de Touraine, il nous semble que cette approche entretient la séparation entre lutte nationale et rapports de classes.

En effet, chez Touraine, les trois thèmes du nationalisme, de la mobilité sociale et du conflit de classes sont associés à des principes d'analyse distincts, à des langages qui représentent chacun un point de vue pour l'analyse des problèmes sociaux et politiques des sociétés dépendantes. De sorte que l'étude pourrait n'adopter qu'une ligne d'analyse et conduire à des résultats intéressants. Cette éventualité est même nécessaire dans certains cas. "Lorsqu'on considère les situations les plus archaïques, plus on s'éloigne de la voie révolutionnaire, plus les forces en présence tendent à s'opposer, ou du moins à se séparer, ce qui conduit à considérer séparément les problèmes de la mobilité, ceux du nationalisme et ceux des classes sociales ${ }^{3}$.» En somme, la

1. Alain Touraine, les Sociétés dépendantes, Paris, Duculot 1976, 266 p., p. 160.

2. Ibid., p. 161.

3. Ibid., p. 195. 
complexité des situations exige de définir les mouvements sociaux comme un système d'action reliant les trois problèmes, mais ces problèmes n'en renvoient pas moins à des principes d'analyse distincts. Dans la problématique tourainienne, luttes nationales et luttes de classes sont juxtaposées les unes aux autres, et non intégrées dans un même système conceptuel.

Cette absence d'articulation analytique entre nation et rapports de classes fait apparaître la lutte de libération nationale comme celle d'une nation s'opposant à une domination étrangère, comme une relation entre nations. Est-ce à dire que la nation est alors définie comme une communauté, possédant des traits culturels homogènes, revendiquant son autonomie politique? Cette conception communautariste exclut la division en classes sociales au sein de la nation. Surtout, elle interdit de penser les effets de la dépendance (ou de l'oppression) sur la structure même des classes et de leurs rapports ${ }^{4}$. Il nous semble que l'oppression nationale doive plutôt être définie comme un aspect des rapports capitalistes d'exploitation et de domination, de sorte que la lutte nationale, tout en conservant sa spécificité, soit considérée comme une forme de luttes de classes. Ainsi, nation et rapports de classes ne constituent plus des principes d'analyse juxtaposés mais ils sont intégrés dans une même problématique.

L'une des difficultés d'utilisation du concept de mouvement social provient d'un flottement entre les deux aspects principaux de sa définition : d'une part, les orientations normatives et d'autre part, les agents sociaux. L'accent est le plus souvent mis sur les orientations normatives définies par les acteurs eux-mêmes et alors l'analyse fait passer au second plan les pratiques réelles des forces sociales en présence et risque de masquer les alliances complexes de classes et fractions de classes qui en sont les agents principaux. La tentation est grande alors de distinguer une série de mouvements sociaux selon la nature de leurs revendications et de leur idéologie.

La notion de mouvement social n'aura d'utilité pour nous que pour désigner un ensemble multiforme et indifférencié de classes ou d'organisations dont le principe d'unité réside soit dans l'identification d'un problème (mouvement urbain, etc.), soit dans certaines caractéristiques des membres (mouvement étudiant, des femmes, etc.).

Une analyse des rapports de classes est plus efficace pour déceler à travers les luttes, à la fois les enjeux contradictoires et les intérêts de classes et les fractions en présence afin d'identifier les formes principales d'organisation des rapports sociaux en ce qui concerne surtout la question nationale. Mais il faut d'abord préciser comment l'oppression nationale constitue une forme de rapports capitalistes d'exploitation et de domination.

\section{OPPRESSION NATIONALE ET RAPPORTS DE CLASSES}

La question centrale est donc celle d'identifier les multiples contradictions dont est traversé le Québec, d'indiquer les relations entre elles, de répérer

4. Dans l'article cité, Touraine fait référence à la domination étrangère externe alors que nous analysons une situation de domination nationale interne à une formation sociale. Mais l'argumentation est la même, à savoir que dépendance et oppression ne doivent pas être analysées comme des phénomènes extérieurs aux rapports de classes. 
celles qui font l'objet de luttes mobilisatrices, c'est-à-dire qui constituent un enjeu. Nous verrons ensuite les effets de ces éléments structurels sur les classes sociales, puis dans la conjoncture, comment s'organisent les rapports sociaux.

L'État québécois, même s'il possède presque toutes les caractéristiques d'un État : Parlement, cour de justice, bureaucratie, etc. et même si par ses propres moyens il contribue à la reproduction des rapports sociaux, n'est en réalité qu'un segment, qu'une unité de l'État canadien, car il ne possède pas tous les pouvoirs qui ont déterminé les formes spécifiques du rapport d'exploitation au Québec. Les principaux mécanismes d'intervention économique relèvent en effet de l'État canadien : politique monétaire et fiscale, tarifs et douanes, banque et crédit, commerce extérieur, etc. L'État provincial du Québec a une juridiction prédominante sur les domaines de l'éducation, de la langue et de la culture, du droit civil et des relations de travail, et partage avec Ottawa la juridiction sur la santé, la police, la taxation, etc. En d'autres termes, les fonctions d'accumulation du capital et de légitimation idéologique sont à prédominance fédérale et provinciale respectivement, celles de reproduction de la force de travail (immigration, éducation, santé), d'intégration (lois du travail, mécanismes de consultation, "tripartisme») et de répression (armée, police, pénitenciers) sont partagées par les deux niveaux de gouvernement. Le Québec, du fait de l'existence d'un État politique provincial, doit alors être analysé comme un lieu de condensation de certains aspects seulement des luttes de classes même si la juridiction sur les relations de travail et la présence d'une partie de la bourgeoisie anglo-canadienne font que beaucoup de conflits apparaissent internes au Québec.

Ce partage des pouvoirs, réalisé au milieu du siècle dernier sous la direction du capital bancaire et marchand anglo-britannique ayant des intérêts surtout dans l'échange commercial et les transports ${ }^{5}$ est le résultat des conditions de la formation de la Confédération. Le régime fédéral en créant une sorte de marché commun des colonies anglaises réunissait des intérêts économiques déjà régionalement diversifiés (producteurs indépendants de l'Ontario, banquiers et industriels de Montréal, petite bourgeoisie francophone du Québec, marchands des Maritimes, etc.) dont l'unité ne pouvait reposer que sur l'accroissement des échanges et l'apport de l'État central à l'industrialisation. Avec le développement d'économies de plus en plus régionales basées sur les richesses naturelles sous juridiction provinciale et liées à l'investissement direct américain, l'unité politique canadienne est demeurée fragile car les nouvelles fractions dominantes régionalement auront davantage besoin de contrôler et de renforcer les États provinciaux de plus en plus puissants vis-à-vis d'Ottawa ${ }^{6}$.

Ainsi, les forces sociales qui ont présidé à l'unification de colonies anglaises constituaient un espace économique marchand par la formation de l'État fédéral canadien. Mais la bourgeoisie marchande et bancaire, dominante au

5. Tom Naylor, "The History of Domestic and Foreign Capital in Canada» et Jim Laxer, "Introduction to the Political Economy of Canada», dans Robert H. Laxer, édit., $\mathrm{Ca}$ nada Ltd., Toronto, McClelland and Stewart, 1973, 271 p.

6. Garth Stevenson, «Federalism and the Political Economy of the Canadian State», dans Leo Panitch, édit., The Canadian State, Political Economy and Political Power, Toronto, University of Toronto Press, 1977, 465 p. 71-100. 
milieu du siècle dernier, a produit elle-même les conditions de son affaiblissement, de sa dépendance et de sa soumission au capital industriel américain en accélérant la pénétration du mode de production capitaliste au Canada. En effet, dès la fin du siècle dernier, l'État central adopte une politique dite nationale qui consiste en une hausse considérable des tarifs sur l'importation des produits manufacturés. Cette politique a en principe pour objectif de favoriser la fabrication au Canada de produits nécessaires à la consommation, et permet effectivement une expansion considérable de l'industrie du textile, du papier et du tabac, et le maintien de l'importance de l'industrie du cuir et du bois. Tous ces secteurs, sauf le tabac, sont encore contrôlés par des capitalistes canadiens. Mais sous la domination de la bourgeoisie marchande davantage intéressée par le commerce avec l'Angleterre, le capital industriel canadien s'était fort peu développé, de sorte que la politique tarifaire a eu pour principal effet la pénétration du capital industriel américain. Les filiales d'entreprises américaines profitent non seulement d'un marché local mais aussi des avantages canadiens consentis pour l'exportation vers les Etats-Unis et l'Angleterre. Dès la fin du siècle dernier, la moitié des mines du Québec et de l'Ontario est contrôlée par des intérêts américains. Aujourd'hui, ces mêmes capitaux contrôlent environ $60 \%$ de toute l'industrie manufacturière canadienne; cette poussée de l'impérialisme américain au Canada fut réalisée en grande partie (près de $90 \%$ selon certaines sources) à l'aide de fonds en provenance même du Canada : profits non distribués des filiales et emprunts sur le marché canadien. En somme, les politiques gouvernementales et le système bancaire ont contribué à la mainmise du capital américain sur l'économie canadienne, mainmise profitable puisque de 1960 à 1969 les Etats-Unis ont rapatrié \$2,6 milliards de plus qu'ils n'avaient investis au Canada?

Trois caractéristiques ressortent clairement de ces quelques notes historiques.

1.- Les rapports capitalistes d'exploitation prennent toujours une forme particulière selon les régions, parce que l'inégale pénétration du capitalisme dans les différentes branches de l'économie se traduit par des concentrations spatiales de certains secteurs industriels. Ainsi, la dissolution des formes anciennes de production agricole familiale ou artisanale est plus ou moins lente selon les régions; de même des branches entières de l'économie où la technologie est moins développée et l'utilisation de la main-d'œuvre plus intensive, comme le textile ou le vêtement, sont concentrées dans certaines régions; enfin, les grandes entreprises monopolistes multi-nationales déconcentrent leurs activités productives et leurs forces de travail en divisant leurs unités de production en centrales et périphériques et en les représentant sur divers territoires selon la qualification et le coût de la main-d'œuvre. Cette forme régionale de l'inégal développement des rapports capitalistes constitue la base objective d'une opposition d'intérêts entre fractions de la bourgeoisie et fractions de la classe ouvrière dans l'ensemble canadien et, à certains moments, d'une alliance entre fractions bourgeoise et ouvrière sur une base régionale. Ainsi ouvriers et patrons de l'industrie du textile du Québec favorisent les tarifs et les quotas à

7. Ne comptons que sur nos propres moyens, Document de la CSN, 1972, et Kari Levitt, la Capitulation tranquille, l'Étincelle, 1972, $220 \mathrm{p}$. 
l'importation alors que les «consommateurs» de l'Ouest préféreraient des prix moins élevés. De même pour le gaz et le pétrole de l'Ouest, le bois de la Colombie, etc.

2- L'inégal développement et la fragmentation régionale de l'économie sont accentués par l'impérialisme américain qui investit dans les secteurs économiques les plus productifs et surtout localisés en Ontario (automobile, chimie, outillage électrique, etc.) d'une part, et dans les industries liées aux ressources naturelles (pétrole de l'Ouest, fer du Québec, etc.) d'autre part. En plus de produire ses effets propres sur la spécialisation régionale, l'impérialisme américain produit ses effets spécifiques de domination sur l'économie canadienne : unités centrales aux États-Unis (sièges sociaux, centres de recherches, produits finis) et unités périphériques au Canada (extraction des matières premières, usines d'assemblage) et donc transfert de plus-value vers les États-Unis ; le développement économique du Canada prend une forme complémentaire à celui des États-Unis et est donc orienté, pour une bonne part, directement par les multinationales américaines.

3- La régionalisation et la dépendance économique expliquent à leur tour la forme politique décentralisée du Canada, les intérêts économiques canadiens et américains s'appuyant sur la concurrence entre les États provinciaux pour favoriser leurs investissements : concessions minières, prix de l'énergie hydroélectrique, subventions directes, etc. Mais en même temps cette forme d'État contribue à maintenir sinon à accentuer les inégalités régionales et la dépendance économique à l'égard des États-Unis, car les États provinciaux sont plus soucieux de leurs intérêts particuliers que de l'unité nationale ou de l'indépendance économique. Il ne faut pas oublier cependant l'importance des forces économiques ayant intérêt à maintenir ou accroître les pouvoirs de l'État central, mais notre propos se limite ici à repérer des sources de tension ou des enjeux autour desquels s'organiseront les luttes de classes.

Reste entier le problème de l'oppression nationale des québécois francophones à l'intérieur du Canada. Si ce qui précède est correct, l'oppression nationale doit présenter des caractères spécifiques et distincts à la fois de l'inégal développement qui balkanise l'économie canadienne, de la dépendance économique envers les États-Unis et de la répartition des pouvoirs entre les États provinciaux et l'État central. Un bref examen de la question nationale au Canada permettra aussi de faire apparaître un autre facette de la forme politique canadienne.

Ici encore, faisons un bref rappel historique qui nous évitera de longs détours théoriques. La Confédération canadienne marquait, avons-nous dit, l'aboutissement des efforts de la bourgeoisie bancaire et commerciale pour former un espace économique marchand autonome vis-à-vis de l'Angleterre et distinct des États-Unis. Cet espace économique inclut le Québec où sont concentrés les francophones et fait de ceux-ci une minorité linguistique soumise aux intérêts marchands par l'intermédiaire de l'État central. Déja l'Union (1840-1867) avait «supprimé le formidable obstacle que représentait le nationalisme québé- 
cois à la croissance économique de l'ancien Haut-Canada ${ }^{8}$ "car la petite bourgeoisie traditionnelle s'opposait au développement des communications EstOuest et à celui du capitalisme commercial, son pouvoir politique étant lié au maintien de la prédominance de l'agriculture'. La présence des francophones (ou ce que d'autres appellent le caractère bi-national du Canada) explique aussi la forme politique décentralisée du Canada et en particulier la juridiction des États provinciaux dans les domaines de la culture, de l'éducation et de la langue. Mais en même temps ce partage consacre et reproduit une oppression nationale que l'on peut définir elle aussi comme une forme particulière que prennent les rapports d'exploitation capitaliste, une forme spécifique de domination de classes $^{10}$.

En effet, la formation d'un État-nation à partir de groupes linguistiques différents suppose déjà l'existence de rapports de classes bien spécifiques et en particulier l'hégémonie d'une fraction bourgeoise d'un groupe linguistique, en l'oscurence celle de la bourgeoisie marchande anglo-canadienne et, plus tard, celle de la bourgoisie industrielle anglo-canadienne. Cette hégémonie implique un processus d'assimilation et de dissolution de toutes les barrières faisant obstacle à la libre circulation des marchandises, du capital et de la force de travail. Pour la bourgeoisie dominante, la langue et la culture constituent des obstacles à l'homogénéisation du territoire, aux communications et à la diffusion de l'idéologie nationale. La légitimation de l'État-nation est sans cesse remise en question par l'existence de deux langues sur le même territoire, de même que l'unité des fractions bourgeoises est plus difficile à réaliser.

l'oppression nationale n'est ni la domination d'une nation sur une autre nation ni la coexistence de deux structures de classes d'appartenance nationale différente. Il s'agit plutôt d'une forme de domination de classes ou de rapports sociaux, forme qui se caractérise par la domination linguistique et culturelle, de la même façon que la «domination régionale» est une articulation particulière des rapports de classes et ne peut être définie comme une relation de région à région. Car, la domination linguistique et culturelle en traversant l'ensemble des classes sociales, introduit une dimension particulière dans les relations entre les classes. Plus concrètement, elle se définit par des pratiques de discrimination linguistique et culturelle, mais dont les effets varient selon la place occupée dans les rapports de classes.

Les classes sociales ne sont pas affectées de la même manière par les pratiques discriminatoires. Pour la classe ouvrière, ces pratiques signifient des contraintes spécifiques quant aux conditions de la vente-achat de sa force de travail, de son utilisation productive et de sa reproduction. Les ouvriers francophones subissent ainsi la discrimination sur le marché du travail, dans l'affectation des tâches, la promotion, la mobilité, etc., discrimination qui se traduit concrètement par une surexploitation et des bas salaires. Rappelons que les

8. Noël Vallerand, «Histoire des faits économiques de la vallée du Saint-Laurent (17601866) ", dans Économie québécoise, Montréal, PUQ, 1969, p. 68.

9. Gilles Bourque et Nicole Frenette, « La structure nationale québécoise», dans Socialisme québécois, $\mathrm{n}^{\text {os }}$ 21-22, 1971, p. 109-155.

10. Voir à ce sujet le texte de Carol Levasseur, «Mouvements nationalitaires et structure de domination nationale», Département de sciences politiques, Université Laval, polycopié, 60 pages, automne 1977. 
québécois francophones occupent l'avant-dernier rang de tous les groupes linguistiques dans la hiérarchie des salaires au Québec.

Pour la classe bourgeoise, la discrimination affecte les conditions de mise en valeur et d'accumulation de ses capitaux, de même que ses possibilités d'accès aux postes de haute direction des entreprises. La thèse récente d'Arnaud Sales a montré que l'accès de la bourgeoisie francophone aux ressources financières des banques canadiennes-anglaises était difficile, qu'il existe une correspondance assez stricte entre l'origine de la propriété et l'origine des dirigeants, que les québécois francophones ne contrôlent que $5 \%$ des entreprises du secteur monopoliste situées au Québec ${ }^{11}$.

La place occupée par la petite bourgeoisie dans la division du travail est telle que la discrimination l'affecte tout particulièrement. La petite bourgeoisie se situe en effet du côté du travail intellectuel et sa connaissance de la langue comme sa capacité de communiquer et de s'exprimer sont ses instruments de travail privilégiés. De plus, les fonctions occupées sont souvent de type idéologique (v.g. information, enseignement...) ou politique (v.g. fonctionnaire, direction de personnel dans l'entreprise privée...) et elles exigent une certaine adhésion aux objectifs des classes dominantes. Ainsi s'explique, en partie, la faible importance des québécois francophones dans le fonctionnarisme fédéral et parmi les cadres des entreprises privées.

Si les formes de l'oppression nationale varient selon les classes, il en est de même des pratiques ou luttes contre cette oppression nationale. Lutter contre la surexploitation n'exige pas les mêmes moyens de mobilisation et ne comporte pas les mêmes implications que de lutter pour faire lever certaines contraintes à l'approvisionnement en capitaux. Inversement, il serait intéressant de relever les taux de transferts linguistiques selon les classes et fractions de classes pour mieux apprécier les forces d'assimilation et la capacité de résistance selon la place occupée dans les rapports sociaux.

Ainsi les traits fondamentaux de la formation sociale canadienne sont liés l'un à l'autre, forment un tout, et doivent être analysés comme autant d'effets d'un même rapport social. La dépendance économique vis-à-vis de l'impérialisme américain, l'oppression nationale, les inégalités régionales sont autant de formes concrètes qu'a pris le développement des rapports capitalistes de production au Canada, formes concrètes qui expliquent les tensions et conflits entre l'État central et les États provinciaux, ou mieux, qui expliquent la subordination politique des États provinciaux vis-à-vis de l'État central fédéral. Comme effet de retour, cette subordination politique contribue à maintenir et à reproduire ces différentes formes de rapports sociaux. Il est important de souligner fortement ce point car les diverses explications ont tendance actuellement à confondre l'une ou l'autre de ces formes; par exemple, les luttes nationales sont souvent comprises comme étant l'expression déviée de revendications dont l'enjeu réel est le rattrapage économique du Québec (cf. thèse de l'inégal développement du Canada). Les classes dominantes utilisent ces réductions, comme elles réduisent l'exploitation de la classe ouvrière à des formes particulières et isolées l'une de l'autre : salaires, heures de travail, qualification, sécurité d'emploi, etc.

11. Arnaud Sales, Capital, entreprises et bourgeoisie. La différenciation de la bourgeoisie industrielle au Québec, thèse de doctorat, Université de Paris VII, 1976. 
A.u Québec, ces différentes formes des rapports sociaux entraînent une structuration particulière des classes sociales et une organisation spécifique des luttes. La question nationale y prend d'autant plus d'importance que l'État québécois lui-même, dans les limites de ses pouvoirs, assume une partie de la fonction générale de reproduction des rapports de classes, y compris de l'oppression nationale avec l'appui d'une coalition de francophones et d'anglophones.

\section{EFFETS DE L'OPPRESSION NATIONALE SUR LES CLASSES SOCIALES AU QUÉBEC}

Il est impossible de décrire ici la manière dont les classes sociales sont affectées par ces différentes formes concrètes par lesquelles se matérialisent le rapport fondamental d'exploitation. Il faudrait alors distinguer les effets propres du développement inégal, de l'oppression nationale, etc. sur la configuration de chacune des classes et sur les rapports de classes. Quelques exemples d'effets de l'oppression nationale suffiront pour montrer d'abord qu'il s'agit bien d'un type de domination distinct de celui engendré par le développement inégal, ensuite que cette oppression favorise un certain type d'alliances de classes.

\section{LA NOUVELLE PETITE BOURGEOISIE}

Le développement du capitalisme monopoliste (concentration du capital et développement complexifié des forces productives) a nécessité l'intervention de l'État dans l'économie et accru son rôle politique et idéologique. Ces transformations ont entraîné une modification de la structure des classes sociales en accroissant considérablement les fonctions d'encadrement, de supervision et de direction, tant dans les entreprises de production que dans les services commerciaux et bancaires et les appareils politiques et idéologiques d'État. L'extension de ces fonctions a constitué une base matérielle à l'émergence d'une classe néo-petite bourgeoise composée d'agents intermédiaires du capital. Ces agents n'occupent pas des fonctions de direction d'ensemble du procès de travail et des rapports de production, et ne s'approprient donc pas directement le profit. Leurs tâches de surveillance sont des délégations ou des relais du pouvoir capitaliste, à tous les niveaux : politique, économique ou idéologique. S'ils reçoivent une rémunération supérieure, celle-ci doit être considérée comme une prime de loyauté politique.

Les intérêts de cette classe ne sont donc pas liés à l'appropriation directe du profit comme c'est le cas pour les capitalistes individuels dans le procès général d'accumulation du capital. Ces cadres-surveillants (ingénieurs, techniciens, organisateurs du travail, contremaîtres, spécialistes de la sélection et de l'intégration du personnel, directeurs de services administratifs ou commerciaux, responsables de programmes d'enseignement et de recherche, etc.) dépendent davantage du développement des forces productives et de l'extension des fonctions politico-idéologiques.

Au Québec, où l'économie est contrôlée principalement par les capitalistes anglo-canadiens et américains, où une grande part du pouvoir poli- 
tique relève de l'État central fédéral et où la langue de travail dans ces milieux demeure l'anglais, la nouvelle petite bourgeoisie francophone est ainsi limitée dans le nombre de places qu'elle peut occuper. Elle se trouve en compétition inégale avec les anglophones dont les possibilités de carrière sont plus grandes.

Le rapport de la Commission royale d'enquête sur le bilinguisme et le biculturalisme ${ }^{12}$ avait à ce sujet fourni une analyse fort intéressante en isolant l'effet de l'ethnicité sur la disparité des revenus et sur les différences de carrières entre anglophones et francophones. Pour l'ensemble de la population active du Canada, le rapport souligne l'influence de l'état du développement régional, de la structure professionnelle et du niveau d'instruction dans l'explication des écarts de revenus entre anglophones et francophones. En ajoutant l'effet de facteurs tels l'âge, l'industrie et le sous-emploi, il reste quand même un écart inexpliqué que les auteurs évaluent à $38 \%$ ou $24 \%$ dans la région de Montréal selon les méthodes utilisées et qu'ils attribuent à l'ethnicité : «influence nette de l'origine ethnique, tous les autres facteurs demeurant constants» (p. 63). «Bref, l'ensemble des facteurs susmentionnés explique la majeure partie des écarts que nous avons relevés : il faut donc considérer les éléments liés à l'ethnicité comme secondaires, bien qu'appréciables» (p. 78). En d'autres termes, aux facteurs liés au développement inégal (sous-emploi, niveau d'instruction, structure d'occupation, etc.) il faut ajouter l'oppression nationale (ethnicité) pour rendre compte des disparités de revenus entre anglophones et francophones.

En ce qui concerne la place respective des francophones et des anglophones dans les professions administratives et techniques au Québec, la Commission conclut clairement à l'existence de pratiques discriminatoires qui orientent les francophones vers la fonction publique québécoise ou la pratique privée indépendante. «De tous les domaines d'activité communs aux grandes entreprises, exception faite de la direction, c'est dans le génie, la recherche et le développement que la présence francophone et la place du français sont les plus restreintes. Dans tout notre échantillon, $22 \%$ seulement des salariés affectés à ces activités sont francophones» (p. 516). Il en est de même des francophones spécialistes en administration et en comptabilité qui se dirigent surtout vers les firmes privées ou les services gouvernementaux (p. 526). Enfin, «la présence des scientifiques diplômés d'universités francophones dans les diverses industries offre de nombreuses analogies avec celles des ingénieurs. Au gouvernement provincial et dans les administrations municipales, $85 \%$ des scientifiques sont francophones... Comme les ingénieurs de langue française, ils sont faiblement représentés dans les grandes entreprises minières et manufacturières $(14 \%)$, de même que dans le bâtiment, les transports, et les communications (13\%)» (p. 527).

Comment expliquer ces différences d'emplois et de carrières? Selon la Commission, «il semble irréfutable que certaines entreprises pratiquaient la discrimination» (p. 531) même si elle ajoute, et sans qu'aucune donnée vienne appuyer une telle affirmation, que «de nos jours, la sélection rationnelle a succédé dans une large mesure aux méthodes empiriques et subjectives » (. 531).

12. Rapport de la Commission royale d'enquête sur le bilinguisme et biculturalisme, livre III, le Monde du travail, Ottawa, Imprimeur de la Reine, 1969, p. 19-52 et 61-78. 
À peu de choses près, la même situation prévaut dans l'administration publique fédérale. Alors que les francophones représentent $26 \%$ de la population active au Canada, ils ne sont que $22 \%$ dans l'ensemble de la fonction publique et $14,4 \%$ seulement dans la catégorie spécialistes et techniciens (ingénieurs, scientifiques, juristes, médecins, sociologues, etc.). De plus, les fonctionnaires francophones reçoivent des traitements inférieurs à leurs collègues anglophones ayant le même niveau d'instruction ou appartenant à la même catégorie professionnelle. Fait significatif, l'écart des traitements est le plus considérable dans le cas des diplômés d'université (p. 224-231).

Les effets de l'oppression nationale sont donc évidents pour la petitebourgeoisie dont la langue est le principal instrument de travail. Des observations sur les disparités régionales (taux de chômage, structure industrielle, répartition sectorielle de l'emploi, évolution des investissements) elles-mêmes accentuées par l'impérialisme américain montreraient des effets du même type : bas salaires, postes peu nombreux dans la recherche, etc. Devant ces obstacles, la résistance de la petite bourgeoisie se manifeste par des tentatives pour récupérer les juridictions fédérales et les administrations correspondantes et créer des entreprises d'État afin de compenser la faiblesse de la bourgeoisie québécoise francophone. Le rapatriement des pouvoirs actuellement tenus par l'État central lui permettrait, en plus de multiplier les postes accessibles, d'accroître son champ d'intervention sur les politiques économiques et sur l'inégal développement. La dépendance économique, la subordination politique et l'oppression nationale se conjuguent donc pour assurer une base matérielle à la constitution de la nouvelle petite-bourgeoisie francophone comme force sociale autonome.

Cependant, ces conditions objectives ne conduisent pas mécaniquement à des luttes nationales dans le champ politique. De plus, les divers types de domination peuvent contribuer au fractionnement politique de la petite bourgeoisie car celle-ci est extrêmement dépendante de la bourgeoisie. Les contradictions entre fractions du capital peuvent ainsi se reproduire dans des allégeances politiques différentes au sein même de la petite-bourgeoisie.

\section{L,A BOURGEOISIE}

Nous avons rappelé brièvement plus haut les conditions historiques de la marginalisation de la bourgeoisie québécoise francophone par la bourgeoisie anglo-canadienne et de la pénétration de l'impérialisme américain au Canada et au Québec. La place limitée des bourgeois québécois francophones dans la propriété des entreprises et le déplacement du centre économique de Montréal vers l'Ontario sont des faits connus même si la discussion reste ouverte sur l'importance de facteurs explicatifs tels les changements technologiques, la concentration du capital, les politiques économiques canadiennes et provinciales, et la question nationale. Il semble toutefois possible d'affirmer que l'oppression nationale produit ses effets propres sur les possibilités d'accumulation de la bourgeoisie québécoise francophone.

Ein effet, les travaux récents d'Arnaud Sales montrent que les sources d'approvisionnement en capital des entreprises du Québec auprès des banques 
constituent des réseaux «nationaux» relativement étanches de sorte que les entreprises francophones ont surtout accès au capital bancaire francophone. Or l'actif bancaire francophone représente moins de $10 \%$ du total canadien. Un réseau du même type existe pour le recrutement de la haute direction des entreprises : «l'origine nationale des plus hauts dirigeants est très largement déterminée par l'origine nationale ou ethnique du principal actionnaire ou du groupe qui contrôle l'entreprise ${ }^{13}$. Les chances des francophones d'accéder à la haute direction sont en effet très minimes lorsque l'on sait que le pourcentage des grandes entreprises (c'est-à-dire celles où propriété et direction ne sont pas assurées par le même individu) contrôlées par la bourgeoisie francophone ne représente que 4 à $5 \%$.

Ainsi la bourgeoisie francophone rencontre certains obstacles à son expansion dus à l'ethnicité. Toutefois il ne faut pas en conclure que la bourgeoisie francophone est de ce fait nationaliste. D'une part, certaines entreprises ont réussi à limiter sinon à lever complètement les obstacles à leur accumulation et ainsi à occuper une place importante parmi les grandes entreprises. D'autre part, les intérêts de la bourgeoisie québécoise francophone sont fractionnés politiquement selon les avantages qu'elle peut retirer de la Confédération. Les entreprises dont le marché est canadien et surtout celles qui exportent des produits finis dépendent davantage des politiques tarifaires et commerciales de l'État central ${ }^{14}$. Enfin, il faut aussi se demander si le nationalisme actuel du P.Q. porté surtout par la petite bourgeoisie et appuyé par la classe ouvrière n'est pas susceptible par le mode de gestion des rapports sociaux qu'il veut instaurer, d'effrayer la bourgeoisie.

\section{LA CLASSE OUVRIËRE}

Peu de recherches ont été effectuées sur les effets spécifiques de l'oppression nationale sur les ouvriers et les employés. Nous devrons nous limiter à quelques remarques d'ordre général.

Une première constatation s'impose : le développement inégal du capitalisme crée des disparités de toutes sortes au niveau proprement économique : entre régions et secteurs industriels, et, à l'intérieur de ceux-ci, entre degrés de concentration des entreprises. Ces disparités liées à des taux d'exploitation différents et à des rythmes différents d'accumulation du capital sont une des raisons principales de la non-homogénéité des luttes de la classe ouvrière : des secteurs industriels sont en crise alors que d'autres connaissent une forte expansion, les conditions de travail et de salaires varient d'une entreprise ou d'un secteur à l'autre, etc. La mobilisation et les luttes se trouvent pour ainsi dire étalées dans le temps.

A cela s'ajoutent les divisions produites par la dépendance, la subordination et l'oppression. En premier lieu, la dualité des juridictions politiques constitue un facteur de division dè la classe ouvrière canadienne. Si certaines luttes

13. Arnaud Sales, «La question linguistique et les directions d'entreprises», le Devoir, 27 avril 1977. Voir aussi pour l'ensemble de la question, Arnaud Sales, thèse de doctorat, op. cit.

14. Garth Stevenson, op. cit. 
d'opposition politique peuvent mobiliser l'ensemble de la classe ouvrière canadienne comme dans le cas des mesures fédérales anti-inflationnistes, par contre des luttes «provinciales» sont alors menées s'il s'agit de négociations dans les institutions publiques de santé ou d'éducation ou encore s'il s'agit de résister à la répression exercée par la limitation des droits syndicaux ou des activités politiques.

En second lieu, les positions de la classe ouvrière canadienne-anglaise face à la question nationale au Québec sont une source de division avec la classe ouvrière québécoise francophone. On peut retenir comme indices à cet effet les prises de position des partis comme le Parti communiste canadien ou le Nouveau Parti démocratique qui n'ont jamais reconnu la légitimité de l'autonomie politique du Québec. En troisième lieu, la classe ouvrière québécoise est divisée sur une base culturelle et linguistique. «Il serait plus juste de dire que la division linguistique et culturelle entre ouvriers anglophones et ouvriers francophones tend fortement à reproduire les clivages entre «aristocratie ouvrière» de travailleurs hautement qualifiés et travailleurs de base. C'est un fait, les travailleurs francophones sont relativement peu qualifiés et la déqualification croissante du travail industriel les atteint sensiblement plus, dans leur masse, que l'aristocratie ouvrière principalement anglophone... La question nationale se pose donc pour la classe ouvrière, elle se pose sur le terrain de la division sociale du travail à l'intérieur des rapports de production capitaliste ${ }^{15}$.» On pourrait faire l'hypothèse que ces différences de qualification sont entretenues par les rapports politiques à l'intérieur des entreprises contrôlées par les anglophones : par la sélection, la promotion et autres avantages de salaires ou de conditions de travail, les ouvriers anglophones seraient constamment favorisés au détriment des francophones. Les ouvriers francophones subissent ainsi une discrimination culturelle et linguistique de la part des anglophones en plus d'être soumis à la répression de leur langue et culture «populaires» par la bourgeoisie francophone.

Nous avons soutenu jusqu'ici :

a) que l'oppression nationale fait partie de la structure même des rapports capitalistes au Canada car l'unité canadienne s'est constituée sous l'hégémonie de la bourgeoisie anglo-canadienne;

b) que l'oppression nationale comme forme concrète de ces rapports se définit par la discrimination linguistique et culturelle et se distingue de la «domination régionale» reliée à l'inégal développement;

c) que les effets de l'oppression nationale varient selon la place occupée dans les rapports de production et de ce fait contribuent au façonnement des rapports de classes.

15. Michel Van Schendel, "Notes : dépendance et autonomies politiques de la classe ouvrière", dans Contradictions, $\mathrm{n}^{\circ} 3$, janvier-juin 1973, p. 53-102, 93-94. 


\section{IV. ÉLÉMENTS DE LA CONJONCTURE SOCIO-ÉCONOMIQUE ET POLITIQUE DU QUÉBEC}

Avant d'aborder directement l'analyse de la conjoncture québécoise, il est nécessaire de relever les orientations théoriques qui ont marqué les analyses récentes du Québec. D'une part, certains analystes marxistes du Québec n'auront opéré qu'un transfert simpliste marqué d'idéalisme en appliquant le concept de formation sociale à la société québécoise. La proposition ainsi formulée ne faisait que prolonger la voie nationaliste d'analyse du Québec en accordant une place déterminante aux caractéristiques culturelles (culture, langue, mode de vie, idéologies spécifiques) dans la désignation du Québec comme formation sociale. Ils reprenaient ainsi l'historiographie traditionnelle pour laquelle 1'histoire du Québec n'est que celle d'une nation opprimée par les anglo-saxons. Cette insistance sur les caractéristiques culturelles a conduit à réduire l'importance des caractéristiques économiques et politiques et à négliger tous les facteurs explicatifs liés au développement du capitalisme canadien et à l'impérialisme américain et dont le Québec est partie intégrante. S'oppose à cette approche de définition de la société québécoise comme formation sociale, l'hypothèse qui définit le Québec comme une région du Canada dont la spécificité provient du développement inégal de l'économie canadienne. La question nationale devient alors subordonnée à celle de l'économie et elle n'est ni plus ni moins qu'un effet de la structure économique du Canada. La base nationale, les caractéristiques culturelles et linguistiques ainsi que la structure politique fédérale-provinciale sont considérées comme secondaires et n'apparaissent pour ainsi dire pas dans l'analyse de la question nationale du Québec. La dynamique de l'histoire du Québec s'explique donc, suivant cette hypothèse, en regard de la conjoncture économique canadienne et américaine, l'oppression nationale intérieure renvoyant à celle du développement inégal de l'économie.

Quant à nous, face à ces deux orientations théoriques, nous pensons qu'il faille définir le Québec comme un lieu d'articulation spécifique de la question nationale et de la lutte des classes.

Dans le présent texte, nous procédons à une analyse des rapports sociaux internes au Québec, bien que nous soyons conscients qu'il faille la compléter ultérieurement par une étude des rapports sociaux dans l'ensemble canadien.

Dans notre analyse, nous allons nous concentrer davantage sur la période des années 1960-1977 et réfléchir sur quelques aspects de cette conjoncture qui nous permettront d'aborder la discussion d'un certain nombre de questions sociologiques et politiques relatives au développement du Québec. Nous allons subdiviser la période étudiée en trois sous-périodes pour mieux en cerner les caractéristiques : 1) $1960-1966$; 2) 1966-1976; 3) 1976-1977.

\section{LA REVOLUTION TRANQUILLE 1960-1966}

Les années 60 ont été marquées par un réalignement des diverses fractions bourgeoises au Québec. Il nous faut examiner quels sont les rapports qui existent entre les intérêts de chacune de ces fractions et le projet «nationaliste» du gouvernement Lesage. La lutte de la bourgeoisie anglo-canadienne contre le 
nationalisme québécois traditionnel converge dans les années 60 avec celles de la bourgeoisie et de la nouvelle petite bourgeoisie québécoises. Le gouvernement libéral issu de ces luttes procède donc à une modernisation et à une extension de l'appareil d'État et à la mise en place de politiques économiques pouvant permettre, du moins à court terme, à la bourgeoisie anglo-canadienne et à la nouvelle petite bourgeoisie d'y trouver leurs intérêts. Quant à la bourgeoisie américaine, elle est peu menacée dans son intervention au Québec par cette nouvelle politique d'apparence nationaliste. Par conséquent, il n'y aura donc eu aucun réaménagement majeur des places occupées par les différentes bourgeoisies pendant la «révolution tranquille».

Rappelons quelques éléments de cette histoire de la Révolution tranquille et de la période qui l'a précédée.

Pendant la période de la «grande noirceur» soit de 1944 à 1960 où le clergé dirigeait la plupart des institutions scolaires et de santé et où le premier ministre Duplessis prétendait garder le contrôle du développement du Québec en brandissant sa politique autonomiste, pendant ce temps, l'investissement américain entrait massivement au Canada et au Québec. Jusqu'en 1957, les gouvernements libéraux au Canada se succèdent avec le même objectif : le plein emploi. Le gouvernement d'Ottawa devient de plus en plus centralisateur et s'allie directement à l'entreprise privée, source d'emploi. La politique du continentalisme (USA-CANADA) aura des conséquences que les gouvernements n'entreverront que dans les années 1960. La politique fédérale de développement économique produira des disparités régionales aiguës sur lesquelles on tentera d'intervenir dans les années 1960, notamment au Québec. Ce sera là une des bases d'intervention de cet «État de la révolution tranquille». Jusqu'en 1960, l'État n'intervient à peu près pas dans l'économie. Le Québec connaît particulièrement un développement inégal de ses régions; entre 1941 et 1960 , la main-d'œuvre agricole diminue de moitié et celle du secteur tertiaire double ses effectifs. Ce gonflenent du secteur tertiaire ne doit pas être interprété comme un indice de développement mais traduit le sous-développement du secteur secondaire propre au Québec : les USA achetant nos matières premières, les transportant chez eux pour la transformation et nous les revendant comme produits finis. C'est lia un processus caractéristique de l'exploitation économique sous l'impérialisme américain. Pendant cette même période, se développent au Québec quelques industries liées à la transformation des ressources naturelles : cela permet aussi de tirer profit des inégalités salariales entre le Québec et les USA.

L'État conservateur québécois et son allié le clergé catholique ont maintenu le peuple dans une dépendance et une soumission politique et idéologique. Seuls quelques conflits ouvriers permettent à la classe ouvrière de saisir le caractère incompatible de ses intérêts avec ceux que représentent l'État, le haut clergé et la bourgeoisie industrielle. Par ailleurs des intellectuels dénoncent la répression, l'atteinte aux libertés, et inscrivent leurs revendications dans le cadre du progrès tel que tracé par les U.S.A. Il s'agit d'imiter les USA, de moderniser la société, de s'ajuster aux tendances du progrès économique. C'est dans ce cadre que s'inscrit la révolution tranquille des années 1960 inaugurée par la prise du pouvoir au Québec du Parti libéral québécois. 
Nous empruntons la définition de ce qu'a été essentiellement la révolution tranquille à la préface signée Jean-Marc Piotte du livre les Travailleurs contre l'État bourgeois ${ }^{16}$.

La révolution tranquille ne consiste donc pas en une transformation quantitative ou qualitative de la structure économique du Québec, même si elle repose sur une phase cyclique d'expansion du système capitaliste. La Révolution tranquille se résume essentiellement en une transformation des superstructures politiques et idéologiques afin de les adapter au stade économique atteint par le capitalisme, soit le capitalisme monopoliste d'État, transformation que les sociologues bourgeois qualifieront de « rattrapage» ou de «modernisation».

Cette transformation des superstructures s'effectue fondamentalement aux niveaux du rôle économique de l'État et de son rôle dans la formation et l'entretien de la main-d'œuvre. D'une part, l'État passe d'une conception du «laissez-faire» s'inspirant d'un libéralisme dépassé, à une volonté de rationaliser et de régulariser le développement du système capitaliste, tout cela enjolivé et masqué sous le couvert du nationalisme (nationalisation de l'électricité, mise sur pied d'une série d'instruments économiques : S.G.F., S.O.Q.U.E.M., Caisse de dépôt et de placements), instruments dont l'objectif réel est d'améliorer le fonctionnement du système capitaliste et dont la conséquence est la consolidation de la domination de l'impérialisme américain sur le Québec.

D'autre part, pour avoir une main-d'cuvre en meilleure santé et mieux formée, l'État réorganise complètement l'appareil de santé (loi de l'assurance hospitalisation, 1961 et loi des hôpitaux, 1962) et l'appareil scolaire (création du ministère de l'Éducation, 1964 et application des principales recommandations du Rapport Parent).

Ces réponses de l'appareil scolaire et de l'appareil de santé enlèveront au clergé le pouvoir qu'il exerçait sur les écoles et les hôpitaux pour le remettre aux mains de l'État. Cela aura comme conséquence la baisse du prestige du clergé, la substitution de l'idéologie technocratique et l'accroissement du phénomène de désengagement religieux, particulièrement à Montréal où le taux de pratique baissera de $60 \%$ à $30 \%$.

Parallèlement, comme nous l'avons mentionné précédemment, le secteur tertiaire continue de se développer et les secteurs public et para-public augmentent leurs effectifs. L'indice de l'emploi dans les services passe, entre 1961 et 1969, de 100 à 165. Le taux de syndicalisation augmente de $7 \%$ de 1961 à 1967, augmentation due à la syndicalisation du secteur du commerce et du secteur public et para-public.

L'extension de l'appareil d'État qui correspond à l'élargissement des secteurs d'intervention de l'État engendre la formation d'une nouvelle petitebourgeoisie et produit la plate-forme sur laquelle elle asseoira son pouvoir et à partir de laquelle elle s'insérera dans les rapports sociaux dominants.

$\mathrm{Au}$ Québec, c'est par une lutte contre la petite bourgeoisie traditionnelle, représentée dans l'appareil scolaire par le clergé, qu'une nouvelle petite-bourgeoisie composée principalement de laïcs et de technocrates prendra la direction de l'appareil administratif d'État et des appareils idéologiques (école, santé, loisir, culture, information...).

Les premières années de la Révolution tranquille sont marquées par une lutte de la nouvelle petite-bourgeoisie en vue d'occuper les places dans l'appa-

16. J.-M. Piotte, les Travailleurs contre l'État bourgeois, l'Aurore, 1975, 275 pages, p. 19-20. 
reil d'État et d'en arriver à un partage du pouvoir avec la fraction hégémonique de la classe dominante, en l'occurence la bourgeoisie des monopoles. Pour cela, elle tente de s'approprier certains fiefs d'exercice du pouvoir, dont l'école.

C'est là une caractéristique de cette période des débuts de la Révolution tranquille : pendant que les agents de la nouvelle petite bourgeoisie occupent les places de direction et d'organisation dans les appareils d'État comme la santé, l'école et les loisirs, la bourgeoisie anglo-canadienne et américaine lutte pour conquérir l'hégémonie dans le contrôle du développement économique. On assiste pendant une période très brève à un partage des pouvoirs entre les bourgeoisies et la nouvelle petite-bourgeoisie ou du moins, à une phase de relative autonomie de la nouvelle petite-bourgeoisie dans les appareils d'États mentionnés précédemment.

Nous assistons pendant cette période au développement d'institutions de participation des corps intermédiaires. Cette extension de l'exercice de la démocratie par le biais de la participation à des organismes de consultation permettra à la nouvelle petite bourgeoisie pendant cette période de 1960-1966 de partager, sur une base très fragile, le pouvoir avec la bourgeoisie ainsi que le contrôle politique de l'aire québécoise du développement économique et social. La petite bourgeoisie nouvelle s'installe sur des terrains où elle n'est pas susceptible de rencontrer la bourgeoisie, à court terme du moins.

Nous proposons d'analyser cette période non pas uniquement comme un «moment» d'ajustement de l'appareil d'État et de transformation de la rationalité aux objectifs du développement économique mais aussi comme un «moment» de la lutte des classes où non seulement une nouvelle petite bourgeoisie s'affronte à la petite bourgeoisie traditionnelle pour le contrôle de l'appareil d'État mais aussi où temporairement une alliance se forme entre cette nouvelle petite bougeoisie, la bourgeoisie anglo-canadienne ayant des intérêts au Québec et la bourgeoisie francophone.

La révolution tranquille constitue une période pendant laquelle l'alliance politique entre la bourgeoisie anglo-canadienne et la bourgeoisie américaine est marquée de fragilité et de vulnérabilité.

Pour rendre compte de ce qu'a été la «révolution tranquille», il est donc nécessaire de recourir à une analyse complexe des rapports entre les fractions de la classe bourgeoise et ses alliés. Aux alliés déjà mentionnés, il faut ajouter que pendarit cette période l'appareil syndical s'est souvent rallié à la nouvelle petitebourgeoisie et aux fractions de la bourgeoisie (francophone et anglo-saxonne) ayant des intérêts au Québec en appuyant les orientations du projet de développement axé sur le rattrapage économique et la modernisation de l'appareil d'État québécois. Cet appui s'explique du fait que plusieurs réformes mises en place par le Gouvernement Lesage, telles que la démocratisation de l'école et les politiques concernant la santé, peuvent être considérées comme l'aboutissement partiel des revendications des travailleurs depuis les années $\mathbf{5 0 .}$

Si les places occupées par les diverses bourgeoisies ne se sont pas modifiées fondamentalement au cours de cette période, il faut quand même relever que le Québec a été un lieu d'affrontements entre elles; pour l'une il s'agissait de reconquérir une place importante dans le champ économique : ce fut le cas 
de la bourgeoisie anglo-canadienne à qui Duplessis avait fait une lutte sans merci pendant 16 ans; pour les autres, il s'agissait de consolider une place déjà conquise : ce fut le cas de la bourgeoisie américaine et de la bourgeoisie francophone. Enfin, de nouveaux groupes sociaux regroupés sous la nouvelle petite bourgeoisie allaient s'assurer le contrôle de nouvelles places créées par la transformation de l'appareil d'État et déloger peu à peu la petite bourgeoisie traditionnelle. Il y a donc lieu de reconnaitre que les bourgeoisies sont intervenues dans les champs économique et politique sur une base nationale. Cependant, les alliances varieront selon les sous-périodes conjoncturelles des années 60-77 et seule une analyse serrée des intérêts en jeu nous permettrait de cerner les causes de ces variations. Nous ne faisons que suggérer ici le profil d'une grille d'analyse, les limites de notre recherche et de notre réflexion ne nous permettant pas d'aller plus loin pour l'instant. Par exemple, il nous apparaît important d'analyser de plus près comment la croissance de l'appareil administratif d'État et des appareils idéologiques qui lui sont rattachés, a permis la constitution d'une base matérielle permettant l'autonomisation politique de la nouvelle petite-bourgeoisie. Par ailleurs, d'autres données nous semblent importantes à compiler et à analyser pour être en mesure de rendre compte des luttes entre les bourgeoisies à l'intérieur de chacun des secteurs : financier, industriel et commercial. Cela permettrait de dresser une carte d'implantation des bourgeoisies et de voir à l'œuvre le processus de constitution des monopoles canadiens et américains.

LA FIN DE LA RÉVOLUTION TRANQUILLE ET LA MAINMISE POLITIQUE DU CAPITALISME MONOPOLISTE SUR LE QUÉBEC : 1966-1976

L'alliance n'aura pas duré longtemps. Dès 1963, la bourgeoisie anglo-canadienne commence à attaquer la place prise par la petite-bourgeoisie pour accrốtre son champ d'activité. De 1966 à 1970, elle aura l'initiative et elle réussira en 1970 à évincer les fractions nationalistes de la petite-bourgeoisie et de la bourgeoisie québécoises de la direction politique de l'État québécois. C'est à cette date que le Parti libéral dirigé par Robert Bourassa reprendra le pouvoir (perdu en 1966).

Cette période marque aussi l'impossibilité dans un État capitaliste au stade monopoliste, d'une telle forme de distribution du pouvoir, d'un partage du pouvoir entre la fraction hégémonique que représentent les monopoles et la fraction régnante que recouvre la bourgeoisie francophone et la nouvelle petite bourgeoisie anti-monopolistes. La phase de libéralisation est donc suivie après 1966, d'une phase de répression qui coïncide avec les débuts d'une récession. La petite bourgeoisie étatique doit se soumettre et se rallier à la direction hégémonique de la bourgeoisie monopoliste. Il y a élimination dans le bloc au pouvoir de la petite bourgeoisie nouvelle, radicalisation de la bourgeoisie dans son ensemble, radicalisation qui entraîne un accroissement de la répression sur la classe ouvrière. Ainsi l'enjeu se déplace : au rattrapage économique de la révolution tranquille succède une période où les luttes de classes opposent plus directement la classe ouvrière à la bourgeoisie sur le terrain de l'exploitation et de la domination politique et idéologique. 
Sur le plan économique, l'inflation qui s'était maintenue en bas de $3 \%$ jusqu'en 1964, augmentera continuellement à partir de 1965, sauf pour l'année 1970, où l'on observe une baisse. De 1960 à 1966, il y a baisse du chômage de $9,2 \%$ à $4,7 \%$; à partir de 1967 , il y aura croissance régulière et le taux passera de 5,3\% à 8,3\% en 1972 pour atteindre $11,3 \%$ en 1977 .

Par ailleurs, une très grande partie de la main-d'œuvre composée d'environ 2500000 travailleurs doit maintenant se recycler trois fois en cours de carrière; des 150000 chômeurs, $40 \%$ ont entre 18 et 24 ans et leur niveau d'éducation est plus élevé que celui de la moyenne. À part les chômeurs, il faut compter les 68000 assistés sociaux qui dépendent entièrement de l'État pour leur survie économique.

En 1967, le système capitaliste est carrément entré dans une phase de crise, dûe à la conjonction de l'inflation et de la récession. Alors que pendant la Révolution tranquille, l'aile parlementaire québécoise de la bourgeoisie prend des couleurs réformistes à cause de son jeu d'alliances avec la petite bourgeoisie et de l'appui d'une fraction de la classe ouvrière, elle apparaîtra aux commandes de la répression pendant cette phase de la récession. Cette répression ira en s'accentuant et engendrera un durcissement du pouvoir d'État tel qu'il permettra à certains de parler de fascisation du régime politique. Quatre indices principaux sont retenus pour décrire ce processus de fascisation : 1) diminution des dépenses de l'État non directement rentables au profit de l'aide directe ou indirecte à l'entreprise privée; 2) durcissement de la bourgeoisie face aux syndicats dans l'entreprise privée ; 3 ) accroissement du contrôle de la bourgeoisie sur les appareils idéologiques d'État; 4) renforcement des appareils répressifs d'État, notamment de l'appareil policier tant fédéral que provincial $^{17}$.

En 1967, l'aile nationaliste du Parti libéral dirigé par René Lévesque quitte le Parti et fonde un mouvement politique indépendantiste qui deviendra un an plus tard, le Parti québécois.

Dans la période dite de la «révolution tranquille», on a pu parler de collaboration et d'appui au gouvernement libéral de la part des syndicats ouvriers, des groupes étudiants et de la petite-bourgeoisie nationaliste. En 19661967 , cette collaboration se transforme en luttes et pour la première fois depuis 1960, le nombre de conflits ouvriers au Québec dépasse celui de l'Ontario (en Ontario, la main-d'œuvre syndicale est supérieure de 200000 à celle du Québec). En 1967, 145226 travailleurs sont en cause dans des grèves et lock-out et l'on compte 1760950 jours perdus. Utilisent le droit de grève, de nouvelles catégories de travailleurs qui viennent tout juste de l'obtenir, soit ceux des secteurs public et para-public; ils constituent $60 \%$ des travailleurs qui déclenchent la grève ou subissent des lock-out entre 1965 et 1971. Les luttes dans le secteur public entraînent le gouvernement libéral, d'abord, puis celui de l'Union nationale à durcir leurs positions. L'État utilise son appareil judiciaire contre les employés du secteur public : après avoir donné le droit de grève à ses employés, il tente de le lui enlever ou plutôt de restreindre les conditions d'utilisation de ce droit. C'est le conflit entre l'État-législateur et l'État-patron qui 
heurte les syndiqués du secteur public de plein front et qui les amène à questionner la fonction politique de l'État. C'est là le chemin de radicalisation et de politisation des centrales syndicales; les syndiqués et les cadres des organisations syndicales prennent conscience au travers des négociations que l'État est lié aux intérêts de l'entreprise privée qui fixent la latitude de son autonomie.

Cette radicalisation des organisations syndicales et du mouvement ouvrier en général ira en s'accroissant et se précisera dans les années qui suivront 1970; ceci s'explique par le fait que si, en 1960, des intérêts communs ont permis une alliance bourgeoisie-petite-bourgeoisie, on remarque que dès 1966-1967, la coalition s'avère un échec et conduit une fraction importante de la petitebourgeoisie déçue à former son propre parti, le P.Q.; par ailleurs en 1970 et après, la bourgeoisie des monopoles apparaît sans détour sur la scène politique et tente d'imposer ses objectifs de développement économique et d'assujettir aux impératifs de sa rationalité économique, les appareils idéologiques tels que l'école et la santé. Une fraction de la bourgeoisie est évincée et menacée par le développement des monopoles au Québec et au Canada : soit la fraction industrielle et commerciale rattachée au stade concurrentiel du capitalisme, possédant peu de capitaux et contrôlant un marché restreint pour ne pas dire local et incapable de suivre le rythme d'accumulation imposé par le développement du capitalisme au Canada. Cette fraction de la bourgeoisie se rallie au mouvement nationaliste et une partie gagne les rangs du Parti québécois alors qu'une autre joint les rangs de l'Union Nationale. Le passage du stade concurrentiel au stade monopoliste transforme les conditions de développement des forces productives, imposant aux unités industrielles et commerciales plus faibles de s'asservir aux plus grosses ou de disparaitre. Au stade concurrentiel, la concentration s'effectue de telle sorte que soient préservés les intérêts des propriétaires de petites entreprises en autorisant la reconversion du capital dans d'autres secteurs d'activités; au stade monopoliste, la liquidation des petites et moyennes entreprises est une des conditions nécessaires au développement des monopoles. $\mathrm{Au}$ Québec, la disparition des petits agriculteurs est maintenant suivie par la liquidation des petites et moyennes entreprises.

C'est dans le contexte de ce double mouvement : durcissement de l'État québécois / radicalisation du mouvement syndical et des luttes des travailleurs que s'inscriront les élections du 15 novembre 1976. Le Parti québécois prend le pouvoir et apparaît comme le parti porteur des solutions à la crise économique et politique.

\section{LA PRISE DU POUVOIR PAR LE PARTI QUÉBÉCOIS : 1976-1977}

Porté au pouvoir depuis bientôt deux ans, le Parti québécois a reçu un appui électoral non seulement d'une petite-bourgeoisie et d'une bourgeoisie nationalistes mais aussi d'un pourcentage élevé d'ouvriers et d'employés tant à Montréal que dans les autres régions de la province. La seule opposition véritable rencontrée par le P.Q. lors de son élection est venue des partis fédéralistes, tandis que le P.Q. mettait de l'avant son programme de gestion écono- 
mique et sociale. Quant au vote exprimé, il s'appuie sur des motifs politiques qui varient selon les couches sociales auxquelles appartiennent les votants. C'est ainsi que les travailleurs appuieront dans le projet péquiste son programme économique d'orientation social-démocrate susceptible de résoudre les problèmes suscités par la crise, plutôt que son programme politique, alors que la petite-bourgeoisie s'intéressera davantage à la question de l'indépendance politique et moins aux propositions proprement économiques.

A l'examen du bilan du Parti québécois depuis qu'il est au pouvoir, il est permis d'observer que, loin de se développer en tant que parti des travailleurs, le Parti québécois, sous le couvert du nationalisme, s'aligne sur les intérêts de la petite bourgeoisie, et de la fraction de la bourgeoisie québécoise dont les intérêts économiques sont menacés par le développement du capitalisme monopoliste canadien. Le nationalisme est donc le mode d'entrée dans la lutte des classes de ces deux classes sociales. Ce qui ne veut pas dire que cette bourgeoisie non monopoliste soit ardemment nationaliste. Elle l'est conjonctuellement parce que le projet économique péquiste semble présenter des propositions économiques qui vont dans le sens de ses intérêts. Ce qu'elle recherche avant tout c'est l'établissement et la consolidation d'un rythme de production et d'un marché de distribution qui ne soient pas entravés par l'expansion des monopoles. La politique nationaliste pouvant servir de support à la réalisation de leurs intérêts, des propriétaires et entrepreneurs industriels se retrouvent parmi les alliés du Parti québécois. Cependant, il n'est pas rare de voir quelques-uns d'entre eux s'implanter en Ontario et tenter de se développer suivant l'axe Québec-Ontario.

Il n'en va pas de même des couches intellectuelles de la petite bourgeoisie poulr qui le projet péquiste est rattaché directement à ce qu'elles représentent fondamentalement, soit la culture et la langue québécoises. En tant que producteurs et reproducteurs de la culture québécoise, les travailleurs intellectuels trouvent dans la sauvagarde de cette spécificité culturelle et linguistique, leur raison d'être y compris leur survie matérielle. On les retrouve massivement parmi les militants péquistes, appuyant sans réserve l'indépendance du Québec. Il en va de même pour les employés de la bureaucratie d'État et certains cadres de l'entreprise privée.

Face au projet politique du Parti québécois, le mouvement syndical demeure ambivalent. Étant donné le reflet social-démocrate du programme péquiste et la place donnée à la question nationale dans la phase pré-électorale, les travailleurs ont appuyé le Parti québécois. La crise économique s'accentuant, l'inflation montant à une allure vertigineuse depuis 1973-1974, le Parti libéral affaibli ne propose aucun moyen pour alléger les retombées de cette crise sur les travailleurs. Avec l'arrivée du P.Q. sur la scène électorale, les travailleurs croient, ou du moins espèrent, que celui-ci pourra gérer la crise et, par des lois sociales et des modifications au Code du travail, améliorer leurs conditions de vie et établir un rapport de forces dans les conflits de travail qui leur soit plus favorable.

Dans les organisations syndicales, l'arrivée du Parti québécois sur la scène politique a suscité des réactions assez semblables : 
a) La Centrale des syndicats démocratiques, issue d'une scission avec la Centrale des syndicats nationaux en 1972 a tenté d'établir une politique de collaboration avec le Parti libéral dirigé alors par Robert Bourassa. Depuis la prise du pouvoir par le Parti québécois, la C.S.D. n'a pas émis de position claire concernant le Parti et s'est contentée d'appuyer certains projets de loi comme la loi 45 concernant la réforme du Code du travail.

b) La Fédération des travailleurs du Québec dont la filiation remonte aux Unions internationales américaines et qui regroupe la majorité des ouvriers syndiqués au Québec comporte un courant fortement péquiste qui s'exprime ouvertement chez les Métallos unis d'Amérique; d'autres branches négocient directement avec le ministre du Travail l'obtention du monopole syndical sur tel chantier de travail : notamment dans la construction. L'appui de la F.T.Q. au P.Q. est aussi un appui à un parti dont la politique en matière de relations ouvrières est marquée par le trade-unionisme. C'est aussi un appui à la politique économique de son programme pouvant permettre une amélioration des conditions de travail. L'orientation travailliste des luttes menées par la F.T.Q. trouve aussi son écho dans le P.Q. Cette orientation pouvant se traduire du côté gouvernemental par des objectifs de consolidation des droits des syndiqués, de rationalisation du développement économique et par l'implantation de mesures de sécurité sociale. Les interventions politiques touchent le secteur de l'économie sans altérer la propriété privée du capital. Du côté syndical, les luttes économiques portent surtout sur les salaires mais aussi sur le contrôle de certains éléments de la situation de travail tels que l'évaluation des tâches, la sécurité d'emploi, l'ancienneté, les conditions hygiéniques de travail. C'est là, ce qui fonde, croyons-nous, la base de l'appui de la F.T.Q. au P.Q. Cependant, au dernier congrès de la Centrale en 1977, la F.T.Q. réitère son appui au P.Q. non sans susciter des débats houleux parmi les délégués et des dissensions entre les syndicats.

c) La Confédération des Syndicats Nationaux, la Centrale d'Enseignement du Québec et quelques membres de la F.T.Q. prônent la mise sur pied d'un parti des travailleurs. Par ailleurs, le discours idéologique de la C.S.N. et surtout celui de la C.E.Q., dont la majorité des syndiqués sont des cols blancs et des travailleurs intellectuels, surprennent par leur ton radical. Les manifestes qu'elles publient, les slogans de mobilisation laissent clairement apparaître le caractère antagoniste des intérêts de la classe ouvrière et de ceux de la bourgeoisie. La C.S.N. et la C.E.Q. demeurent des centrales syndicales dont l'orientation dominante des luttes menées par les travailleurs syndiqués est tradeunioniste. Cependant, elles connaissent depuis quelques années une remise en question radicale de cette orientation. S'y confronte la lutte idéologique de type révolutionnaire. Celle-ci est caractérisée par l'élaboration d'une idéologie prolétarienne autonome dont le but est de combattre l'idéologie bourgeoise diffusée parmi la classe ouvrière. La dominance de cette pratique est idéologique et en l'absence d'un parti de la classe ouvrière, le risque est grand d'une suridéologisation équivalant à une hésitation quant à la tâche politique de construction d'un parti pour prendre le pouvoir d'état. Sur le plan êconomique, les luttes menées en ce sens visent à développer la conscience ouvrière vis-à-vis de l'exploitation capitaliste. Sur le plan politique, tout en se démarquant des luttes 
travaillistes et corporatistes, elles visent à démasquer le caractère de classe de l'Etat. Ces pratiques sont le plus souvent le fait des couches intellectuelles des salariés exécutants. Par ailleurs, le silence est presque complet sur les positions à tenir quant à la question nationale et quant au projet politique du Parti québécois. Les prochains congrès des centrales syndicales devraient connaître des débats décisifs sur cette question.

Il est important de noter que si le mouvement syndical draine la quasitotalité de la mobilisation ouvrière, il n'est pas seul sur ce terrain. De nombreuses organisations populaires se sont mises sur pied pour défendre les intérêts des ouvriers, des chốmeurs et des assistés sociaux. Par ailleurs, les organisations politiques à tendance maoïste et trotskite se multiplient pendant que le Parti communiste du Québec reste stationnaire dans ses effectifs et quasi absent de la scène politique. La rivalité inter-syndicale, la faiblesse des organisations politiques de gauche, l'opportunisme politique du Parti québécois, la place importante occupée par l'idéologie de la contre-culture chez les jeunes, constituent autant de facteurs explicatifs des formes de politisation de la masse des travailleurs et de leur faible conscience de classe.

Si l'analyse que nous venons de présenter à propos de la conjoncture politique des années 60 à 77 au Québec laisse surtout entrevoir l'occupation de la scène politique par les luttes menées par les bourgeoisies monopoliste et non-monopoliste et par la nouvelle petite bourgeoisie surtout nationaliste, il ne faut pas en déduire une absence de la classe ouvrière dans la lutte des classes. Bien que celle-ci, historiquement, ait été encadrée idéologiquement et politiquement par la petite bourgeoisie, ce qui a réduit ses capacités de luttes autonomes dans la lutte des classes, elle n'en demeure pas moins une classe constituée avec ses organisations propres, des luttes spécifiques qui, de jour en jour, s'orientent vers un affrontement de plus en plus dur avec la bourgeoisie et l'État.

La présentation par le Gouvernement péquiste des premières lois ouvrières telles la loi anti-scab a suscité de vives réactions chez les syndicats. C'est un moment clé qui marque les rapports entre les syndicats et le Gouvernement, en ce sens qu'il a permis aux travailleurs d'établir le lien entre le projet de libération nationale et le caractère de classe du gouvernement péquiste. Dans les premiers projets proposés et approuvés par le gouvernement Lévesque, notamment sur le plan des réformes économiques, apparaissent déjà plus clairement les limites à l'intérieur desquelles le processus d'indépendance du peuple québécois va devoir s'échafauder sous la direction du Parti québécois. Le projet de libération nationale proposé est teinté du caractère de classe du gouvernement péquiste et il est de plus en plus marqué par les contradictions qui régissent les rapports de classes au Québec; c'est le développement de ces contradictions qui sera à la base du contenu du projet d'indépendance que nous connaîtrons dans les prochaines années. Les propositions tant au niveau économique, politique et culturel de ce projet seront l'aboutissement du rapport de forces qui s'établira entre les différentes classes de la société québécoise.

Quatre enjeux principaux orientent les luttes de classes au Québec actuellement : 
1) l'oppression nationale, à laquelle correspondent des luttes linguistiques et culturelles axées sur le contrôle du symbolique;

2) le développement inégal de l'économie canadienne qui conduit à des luttes régionales en vue du rattrapage;

3) l'exploitation économique des travailleurs québécois qui provoque des luttes anti-capitalistes de la part notamment des cols bleus et des cols blancs frappés dans tous les aspects de leur vie quotidienne par la montée de l'inflation.

4) la subordination politique de l'État québécois par rapport à l'État central d'Ottawa qui entraîne des luttes autonomistes visant à la décentralisation des pouvoirs politiques. Ce dernier enjeu a été mis de l'avant par le régime Duplessis, puis repris par Lesage et a donné par la suite naissance à un mouvement plus radical qui a pris forme avec le Parti québécois.

Pour résumer l'orientation des luttes menées par les travailleurs dans cette période des années 1960-1977 nous pouvons dire que les luttes idéologiques demeurent principales; que le militantisme se situe d'abord et avant tout au niveau des revendications économiques et que, d'une certaine manière, cette forme de militantisme est exacerbée; et finalement que les luttes au niveau politique mettant en cause le pouvoir d'Etat demeurent marginales.

Ajoutons que chacune des sous-périodes que nous avons étudiée a été marquée par la dominance de l'un ou l'autre des quatre éléments ou enjeux que nous avons mis en relief pour caractériser la conjoncture des années 60-77. Chaque période est marquée par la prise en charge de l'un ou l'autre de ces éléments :

1960-1966 : Le développement inégal est mis en relief par les luttes pour le rattrapage économique, la modernisation de l'appareil d'État et l'extension du pouvoir d'État.

1966-1976 : Le caractère capitaliste du développement économique ressort dans les grèves, les luttes anti-inflationnistes menées par une classe ouvrière radicalisée. L'État en tant qu'État capitaliste est sujet aux attaques des travailleurs organisés.

1976-1977 : L'oppression nationale, la subordination politique et le développment inégal apparaissent davantage liés les uns aux autres tout en demeurant confondus. La centralisation des pouvoirs au gouvernement fédéral est défini comme le frein au développement du Québec.

Un élément demeure absent ou du moins ne ressort pas clairement dans la définition des enjeux de l'ensemble de cette période : l'impérialisme américain. Par ailleurs les rapports qui existent entre l'oppression nationale, le développement inégal et l'exploitation capitaliste, l'impérialisme américain et la subordination politique, rapports qui tissent la spécifité de la société québécoise n'ont pas encore acquis au sein du peuple québécois la transparence nécessaire pour qu'ils deviennent l'élément moteur et le principe organisateur de l'ensemble des luttes sociales au Québec. C'est cependant à ce niveau que l'histoire de la prochaine décennie continuera de se faire et il est à entrevoir que les organisations de travailleurs y joueront un rôle prépondérant pendant que la lutte pour 


\section{l'hégémonie sera plus vive que jamais entre les différentes bourgeoisies et entre les fractions qui les composent.}

\section{RESUME}

Partant d'un bref commentaire critique du concept de mouvement social dans le schéma tourainien, ce qui constitue la première partie de cet article, nous tentons, dans la seconde partie, de mettre en relief l'articulation qui existe entre la lutte nationale et la lutte des classes au Québec. Il s'agit donc d'identifier les multiples contradictions dont est traversé le Québec en procédant d'abord à un rappel historique rapide et en tentant par la suite de définir la place qu'y occupe l'oppression nationale. Nous posons, dans la troisième partie, la questions des effets spécifiques de l'oppression nationale sur la bourgeoisie, la petite-bourgeoisie et la classe ouvrière. Dans la dernière partie, l'analyse de la conjoncture de la période 1960-1977 nous permet de cerner de plus près les rapports de classe et les enjeux qui marquent leur dynamique.

\section{SUMMARY}

The first part of this article is a brief critical commentary of the concept of social movement as elaborated by Touraine; the second part attempts, on the basis of this analysis, to bring out the connection existing between national struggle and class struggle in Quebec. The multiple contradictions present in Quebec are identified by undertaking a short historical overview. An attempt: is then made to define the place occupied by national oppression in these contradictions. In the third part, the question of the specific effects of national oppression on the bourgeoisie, the petite-bourgeoisie and the working class is put forward. In the last part, an analysis of the social, political and economic situation of the period 1960-1977 permits a closer scrutiny of class relationships and the specific elements that mark their dynamics.

\section{RESUMEN}

La prinera parte de este articulo es un breve comentario crítico del concepto de movimiento social dentro del esquema de Touraine, a partir de este intentamos en la segunda parte de mostrar la articulación que existe entre la lucha nacional y la lucha de clases en Quebec. Se trata en consecuencia de identificar las múltiples contradicciones que atraviesa el Quebec, procediendo primeramente a un recuerdo histórico rápido y tratando enseguida de definir el lugar que ocupa la opresión nacional. En la tercera parte planteamos la cuestión de los efectos específicos de la opresión nacional sobre la burgesia, la pequëna burgesia y la clase obrera. En la última parte, el análisis de la conjuntura 1960-1977, nos permite ver de más cerca las relaciones de clase y los confiictos que marcan su dinámica. 